\title{
Constraint-based modeling analysis of the metabolism of two Pelobacter species
}

\author{
Jun Sun ${ }^{1 *}$, Shelley A Haveman ${ }^{2,3}$, Olivia Bui ${ }^{1}$, Tom R Fahland ${ }^{1,4}$, Derek R Lovley ${ }^{2}$
}

\begin{abstract}
Background: Pelobacter species are commonly found in a number of subsurface environments, and are unique members of the Geobacteraceae family. They are phylogenetically intertwined with both Geobacter and Desulfuromonas species. Pelobacter species likely play important roles in the fermentative degradation of unusual organic matters and syntrophic metabolism in the natural environments, and are of interest for applications in bioremediation and microbial fuel cells.

Results: In order to better understand the physiology of Pelobacter species, genome-scale metabolic models for Pelobacter carbinolicus and Pelobacter propionicus were developed. Model development was greatly aided by the availability of models of the closely related Geobacter sulfurreducens and G. metallireducens. The reconstructed $P$. carbinolicus model contains 741 genes and 708 reactions, whereas the reconstructed $P$. propionicus model contains 661 genes and 650 reactions. A total of 470 reactions are shared among the two Pelobacter models and the two Geobacter models. The different reactions between the Pelobacter and Geobacter models reflect some unique metabolic capabilities such as fermentative growth for both Pelobacter species. The reconstructed Pelobacter models were validated by simulating published growth conditions including fermentations, hydrogen production in syntrophic co-culture conditions, hydrogen utilization, and Fe(III) reduction. Simulation results matched well with experimental data and indicated the accuracy of the models.

Conclusions: We have developed genome-scale metabolic models of $P$. carbinolicus and $P$. propionicus. These models of Pelobacter metabolism can now be incorporated into the growing repertoire of genome scale models of the Geobacteraceae family to aid in describing the growth and activity of these organisms in anoxic environments and in the study of their roles and interactions in the subsurface microbial community.
\end{abstract}

\section{Background}

Pelobacter species are commonly found in a number of natural environments, including marine sediments [1], muds [2], soils [3], and hydrocarbon-containing environments [4-6]. They likely play important roles in syntrophic degradation of organic matter in these natural environments and are of interest for applications in bioremediation and microbial fuel cells. Pelobacter species and members of Geobacter, Desulfuromonas and Desulfuromusa genera in the deltaproteobacteria form the monophyletic family Geobacteraceae, which can be divided into two distinct subgroups of the Geobacter and Desulfuromonas clusters [7-9]. The Pelobacter species are phylogenetically intertwined with both clusters:

\footnotetext{
* Correspondence: jsun@genomatica.com

${ }^{1}$ Genomatica Inc., 10520 Wateridge Circle, San Diego, CA, USA

Full list of author information is available at the end of the article
}

Pelobacter carbinolicus is within the Desulfuromonas cluster whereas Pelobacter propionicus is within the Geobacter cluster $[7,9,10]$.

$P$. carbinolicus and $P$. propionicus are both strict anaerobes that have distinct physiologies compared with the Geobacter and Desulfuromonas species. P. carbinolicus was first isolated from marine muds whereas P. propionicus was first isolated from freshwater sediments and sewage sludge [6]. Both can grow by fermentation of 2,3-butanediol and acetoin in which the fermentation products are ethanol and acetate for P. carbinolicus, and propionate and acetate for $P$. propionicus [6]. In addition, both Pelobacter species can grow with ethanol, propanol, or butanol under specific conditions [6].

Like Geobacter and Desulfuromonas species, Pelobacter species have the capacity of using $\mathrm{S}^{0}$ as an electron acceptor $[7,10]$. However, Pelobacter species only incompletely
Ciomed Central

(C) 2010 Sun et al; licensee BioMed Central Ltd. This is an Open Access article distributed under the terms of the Creative Commons Attribution License (http://creativecommons.org/licenses/by/2.0), which permits unrestricted use, distribution, and reproduction in any medium, provided the original work is properly cited. 
oxidize organic substrates with this electron acceptor, in contrast to the ability of Geobacter and Desulfuromonas species to completely oxidize acetate and other organic electron donors to carbon dioxide $[7,10,11]$. For example, $P$. carbinolicus only incompletely oxidizes ethanol to acetate with $S^{0}$ reduction [10].

Unlike Geobacter and Desulfuromonas species, P. carbinolicus lacks most of the $c$-type cytochromes [12], which are essential for optimal electron transfer to $\mathrm{Fe}$ (III) in Geobacter sulfurreducens [13-16]. P. carbinolicus reduces $\mathrm{Fe}(\mathrm{III})$ via an indirect mechanism in which elemental sulfur is reduced to sulfide and the sulfide reduces $\mathrm{Fe}(\mathrm{III})$ with the regeneration of elemental sulfur, contrasting with the direct reduction of Fe(III) for Geobacter species [17]. Furthermore, whereas the Geobacter and Desulfuromonas species can transfer electrons to the anodes of microbial fuel cells to produce current, P. carbinolicus could not [18]. Thus, the Pelobacter species are regarded as primarily fermentative/ syntrophic species, whereas the Geobacter and Desulfuromonas species are primarily respiratory species. Comparative genomic studies have suggested that the common ancestor of Pelobacter, Geobacter, and Desulfuromonas species was a respiratory microorganism and that Pelobacter species evolved to fill fermentative and syntrophic niches (4).

Pelobacter and Geobacter species can be closely associated in subsurface environments $[19,20]$. In microbial fuel cells, a coculture of P. carbinolicus and G. sulfurreducens produced current with ethanol as the fuel, under conditions in which current could not be produced by either microorganism alone [18]. Genome-based experimental and computational techniques offer the possibility of predictive modeling microbial physiology and microbial communities [21]. Genome-based analysis of Geobacter species resulted in two constraint-based genome-scale metabolic models [22,23], which were applied in many studies to accelerate our understanding of Geobacter species and their applications [24-29]. The genome sequence of $P$. carbinolicus DSM2380 and P. propionicus DSM2379 have been completed http:// www.jgi.doe.gov. Because of the similarities and differences in metabolic capabilities between Pelobacter and Geobacter species, in silico modeling of these two Pelobacter species will provide insight into their metabolism. In this report, the developments of genome-scale metabolic models of $P$. carbinolicus and $P$. propionicus are described. These models of Pelobacter metabolism can now be incorporated into the growing repertoire of genome scale models of the Geobacteraceae family to aid in describing the growth and activity of these organisms in anoxic environments and in the study of their roles and interactions in the subsurface microbial community.

\section{Methods}

\section{Strains and culture conditions}

P. carbinolicus DSM 2380 was cultured at $30^{\circ} \mathrm{C}$ under strictly anaerobic conditions as previously described [12]. Fermentative cultures were grown in chemostats in bioreactor at dilution rates of 0.03 to $0.06 \mathrm{~h}^{-1}$ with $5 \mathrm{mM}$ acetoin in the medium. Cell growth on acetoin was monitored by measuring the optical density at $600 \mathrm{~nm}$ with a Genesys 2 spectrophotometer (Spectronic Instruments, Rochester, NY). Cell dry weight was determined gravimetrically after drying at $105^{\circ} \mathrm{C}$ for $24 \mathrm{~h}$.

\section{Analytical measurements}

Concentrations of acetoin, acetate, and ethanol were determined with high-pressure liquid chromatography (HPLC) using an LC-10ATVP HPLC (Shimadzu, Kyoto, Japan) equipped with an Aminex HPX-87 H column (300 by $7.8 \mathrm{~mm}$; Bio-Rad, Hercules, CA), with $8 \mathrm{mM}$ $\mathrm{H}_{2} \mathrm{SO}_{4}$ eluent. Acetate was detected with an SPD-10VP UV detector (Shimadzu, Kyoto, Japan) set at $210 \mathrm{~nm}$. Ethanol and acetoin were quantified with an RID-10A refractive index detector (Shimadzu, Kyoto, Japan). Protein concentrations were determined with the bicinchoninic acid (BCA) method [30] with bovine serum albumin as standard.

\section{Metabolic network reconstruction}

The Pelobacter metabolic networks were reconstructed according to previously published procedures [23]. The reconstruction was carried out in SimPheny $[31,32]$ (Genomatica, Inc., CA) from the annotated open reading frames (ORFs) encoded in the Pelobacter genomes. The Pelobacter genomes and the genomes of several highquality genome-scale metabolic models were analyzed using the sequence similarity search (BLAST), and the BLAST results were utilized to create draft models that served to accelerate the reconstruction of the genomescale metabolic models. The reactions and genes in the draft models were manually reviewed using the gene annotations and the available biochemical and physiological information. Biomass compositions in the published G. sulfurreducens model were used to create the biomass demand reactions in both reconstructed Pelobacter models. The resulting networks were then subjected to the gap filling process to allow biomass formation under physiological growth conditions. For gap filling, simulations were performed to determine if the networks could synthesize every biomass component and the missing reactions in the pathways were identified. These reactions were reviewed for gene association, or added as non-gene associated reactions to enable the formation of biomass by the reconstructed networks under physiological conditions. Experimental data were 
collected from published literature or generated in the laboratory, and were applied to validate model simulation results that predict growth and products under corresponding physiological conditions. Model simulations were also used to generate experimentally testable hypotheses and predictions. The experimental findings accordingly were in turn used to further refine and expand the metabolic models in an iterative process.

The detailed list of genes, reactions, metabolites, and gene-protein-reaction (GPR) associations in the metabolic model are available as additional files.

\section{Determination of energy parameters of the metabolic model}

Energy parameters of the $P$. carbinolicus metabolic model including growth-associated maintenance (GAM) energy and non-growth associated maintenance (nGAM) energy were determined using four sets of experimental data obtained in chemostats of $P$. carbinolicus during acetoin fermentation at dilution rates of 0.03 to $0.06 \mathrm{~h}^{-1}$. The experimental data including acetoin uptake rates and acetate production rates were used as constraints to simulate fermentative growth where the ATP maintenance requirement reaction was selected as the objective function to be maximized. The maximum ATP production rates were calculated and plotted against the dilution rates as described earlier [33]. The linear regression of the plot resulted in an equation:

$$
\mathbf{q}_{\mathrm{ATP}}=\boldsymbol{a} \times \boldsymbol{\mu}+\boldsymbol{b}
$$

where $\mathbf{q}_{\mathrm{ATP}}$ is the ATP production rate and $\boldsymbol{\mu}$ is the dilution rate. The intercept $\boldsymbol{b}$ represents nGAM, the ATP requirement at zero growth, whereas the slope $\boldsymbol{a}$ represents GAM, the ATP requirement for growth. GAM was then incorporated into the biomass demand equation, and nGAM was utilized for all further growth simulations.

For the P. propionicus metabolic model, the same biomass demand equation including GAM was applied. The nGAM was estimated using $P$. propionicus fermentative growth yields with 6 different substrates [6].

\section{In silico analysis of metabolism}

The metabolic capabilities of the Pelobacter models were calculated using flux balance analysis through linear optimization [34] in SimPheny. The simulations resulted in flux values in units of $\mathrm{mmol} / \mathrm{g}$ dry weight $(\mathrm{gdw}) / \mathrm{h}$. All simulations were of anaerobic growth on minimal media, where the following external metabolites were allowed to freely enter and leave the network: $\mathrm{CO}_{2}, \mathrm{H}^{+}$, $\mathrm{H}_{2} \mathrm{O}, \mathrm{K}^{+}, \mathrm{Mg}^{2+}, \mathrm{NH}_{4}{ }^{+}, \mathrm{PO}_{4}{ }^{3-}$, and $\mathrm{SO}_{4}{ }^{2-}$. The electron donors or electron acceptors tested were allowed a maximum uptake rate into the network as specified in the results. All other external metabolites were only allowed to leave the system. To simulate physiological conditions, experimental data were used as constraints for flux optimization. Growth simulations were carried out for: 1) optimal growth where biomass synthesis was selected as the objective function to be maximized with substrate uptake rate fixed; or 2) optimal substrate utilization where the substrate uptake rate was chosen as the objective function to be minimized with the growth rate fixed.

\section{Results and Discussion}

\section{Metabolic network reconstruction}

The $P$. carbinolicus draft model was built with base models including previously published Geobacter sulfurreducens [22], Escherichia coli [34], and Bacillus subtilis [35] models. The P. propionicus draft model was prepared with the above base models plus the completed P. carbinolicus and Geobacter metallireducens [23] models. The origin of reactions in the draft models closely reflected the phylogenetic relationships among the species [7]. For example, reactions in the $P$. propionicus draft model originating from the G. sulfurreducens, $G$. metallireducens, and P. carbinolicus models were $40 \%$, $31 \%$, and $14 \%$, respectively, consistent with the fact that P. propionicus is more closely related to G. sulfurreducens and G. metallireducens than to P. carbinolicus [7].

The reactions and their gene associations in the draft models of $P$. carbinolicus and P. propionicus were evaluated manually based on gene annotations, published biochemical and physiological information and external references as previously described [23]. One important metabolic characteristic of Pelobacter species is their fermentative growth on acetoin [6]. During acetoin fermentation, acetoin is degraded into acetaldehyde and acetyl-CoA by the acetoin:2,6-dichlorophenolindophenol (DCPIP) oxidoreductase [36,37]. The acetoin:DCPIP oxidoreductase was purified and characterized in $P$. carbinolicus [38] and was encoded by the $a c o A, a c o B, a c o C$, and $a c o L$ genes [37]. In the reconstructed Pelobacter networks, the reaction was associated with the $a c o A$ (Pcar_0343 \& Ppro_1131), acoB (Pcar_0344 \& Ppro_1132), acoC (Pcar_0345 \& Ppro_1133), and acoL (Pcar_0347 \& Ppro_1137) genes. In the P. carbinolicus model, the resulting acetaldehyde is reduced to ethanol by alcohol dehydrogenase, whereas acetyl-CoA is converted into acetate by phosphate acetyltransferase and acetate kinase (Pcar_2542 \& Pcar_2543) generating ATP, as described previously [36]. In the $P$. propionicus model, acetaldehyde is oxidized to acetyl-CoA by acetaldehyde CoA dehydrogenase (Ppro_0899 or Ppro_1923), and acetyl-CoA is then converted into propionate in a pathway through pyruvate, oxaloacetate, malate, fumarate, succinate, succinyl-CoA, methylmalonyl-CoA, and 
propionyl-CoA [39]. The key enzyme in this pathway, methylmalonyl-CoA mutase, was associated with genes Ppro_1367 \& Ppro_1368. Reactions and their associated genes for fermentative growth on other substrates were also evaluated. Transportation of neutral molecules such as acetoin, 2,3-butanediol, and other alcohols were assumed to be energy free through reversible diffusion.

P. propionicus ferments both ethanol and lactate to acetate and propionate at an approximately $1: 2$ ratio via methylmalonyl-CoA, but the molar biomass yield with ethanol was less than half of that obtained with lactate $[6,40]$. It was suggested that the different biomass yields were likely due to either a metabolic energy requirement for the ferredoxin-dependent reductive carboxylation of acetyl-CoA to pyruvate, or energy conservation in lactate uptake [40]. Since the metabolic energy requirement for acetyl-CoA carboxylation was not demonstrated with any Geobacteraceae family member [22,23], a lactate proton antiporter was utilized to allow energy conservation in lactate uptake in the reconstructed $P$. propionicus network.

Pelobacter species have been reported to use Fe(III) or $S^{0}$ as an electron acceptor $[7,10]$, but studies with $P$. carbinolicus have suggested that $\mathrm{Fe}(\mathrm{III})$ is reduced indirectly through sulfur reduction, with sulfide serving as an electron shuttle for the reduction of Fe(III) [17]. It was suggested that a low concentration of sulfide favored $\mathrm{Fe}$ (III) reduction without precipitation of ferrous mono- or disulfides in Sulfurospirillum deleyianum [41]. Given the standard reduction potentials for the $\mathrm{Fe}^{3+} / \mathrm{Fe}^{2}$ ${ }^{+}$pair $\left(\mathrm{E}^{\circ}=+771 \mathrm{mV}\right)$ and the $\mathrm{S}^{0} / \mathrm{H}_{2} \mathrm{~S}$ pair $\left(\mathrm{E}^{\circ}=-243\right.$ $\mathrm{mV})$ [42], an extracellular nonenzymatic reaction FE3Rs $\left(2 \mathrm{Fe}^{3+}+\mathrm{HS}^{-} \rightarrow 2 \mathrm{Fe}^{2+}+\mathrm{S}+\mathrm{H}^{+}\right)$was used to model the indirect $\mathrm{Fe}(\mathrm{III})$ reduction. In other bacteria, sulfide is regenerated from elemental sulfur by the membranebound sulfur reductase that contains both hydrogenase and sulfur reductase activities $[43,44]$. The sulfur reductase can use hydrogen as electron donor, or $\mathrm{NAD}(\mathrm{P}) \mathrm{H}$ at a reduced activity [43]. Thus, two sulfur reductase reactions SRE and SRE2 were utilized in the reconstructed $P$. carbinolicus network for sulfur reduction with hydrogen or $\mathrm{NAD}(\mathrm{P}) \mathrm{H}$ as electron donors, although the enzymes catalyzing Fe(III) and $S^{\circ}$ reduction in P. carbinolicus have not been identified experimentally.

The gap filling process identified some missing reactions in the pathways that were added as non-gene associated reactions to enable the reconstructed network to synthesize metabolites for biomass formation. The reconstructed $P$. carbinolicus network contains 37 nongene associated reactions whereas the $P$. propionicus network contains 46 non-gene associated reactions. These non-gene associated reactions are presumptive metabolic functions encoded potentially by unknown genes, and thus will be the subject of further genomic and biochemical investigation in the future.
Recently, a sensitivity analysis in E. coli model [45] indicated that small changes in biomass compositions such as protein, RNA, and lipid did not significantly affect predicted growth rates. Biomass compositions in the published G. sulfurreducens model [22] were used to create the biomass demand reactions in both reconstructed Pelobacter models, as they are closely related to G. sulfurreducens. Sensitivity analysis also indicated minimal changes in simulations by small biomass composition changes in the Pelobacter models (data not shown). Metabolic maps including the biomass demand reactions for the $P$. carbinolicus and $P$. propionicus genome-scale metabolic models were provided (see Additional File 1).

\section{Determination of energy parameters and stoichiometry of the electron transport chain}

The energy parameters of the $P$. carbinolicus metabolic model were determined using four sets of physiological data from $P$. carbinolicus during acetoin fermentation in chemostats producing acetate and ethanol. The gassing required for strict anaerobic growth conditions in chemostats could result in: 1) partial loss of ethanol produced from acetoin fermentation through evaporation; 2) ethanol fermentation to produce acetate and $\mathrm{H}_{2}$, where $\mathrm{H}_{2}$ was removed by gassing to release the feedback inhibition. Therefore, only the experimental acetoin uptake rates and acetate production rates were used to simulate the fermentative growth (Figure 1). The linear correlation of $\mathbf{q}_{\text {ATP }}=97.5 \times \boldsymbol{\mu}+0.844$ has a $R^{2}=$ 0.994 indicating high quality of the results. Therefore, the GAM for the P. carbinolicus model is $97.5 \mathrm{mmol}$ $\mathrm{ATP} / \mathrm{gdw}$ and the nGAM is $0.844 \mathrm{mmol} \mathrm{ATP} / \mathrm{gdw} / \mathrm{h}$.

To determine energy parameters of the $P$. propionicus metabolic model, GAMs from the P. carbinolicus model and the G. sulfurreducens model [22] were used to simulate experimental results. The $P$. propionicus $2,3-$ butanediol fermentation had an optimal growth rate $\boldsymbol{\mu}=$ $0.144 \mathrm{~h}^{-1}[6]$ that was used for simulating P. propionicus fermentative growth with 2,3-butanediol, acetoin, and lactate due to similar growth yields for these fermentations. The growth rates and calculated substrate fluxes based on experimental data [6] were used as constraints in simulations to optimize ATP production. The average of the ATP production rates, representing the nGAM of the $P$. propionicus model, was $2.80 \mathrm{mmol} \mathrm{ATP} / \mathrm{gdw} / \mathrm{h}$ calculated using the GAM of the P. carbinolicus model. Therefore, the GAM for the $P$. propionicus model is $97.5 \mathrm{mmol} \mathrm{ATP} / \mathrm{gdw}$ and the nGAM is $2.80 \mathrm{mmol}$ $\mathrm{ATP} / \mathrm{gdw} / \mathrm{h}$.

The Pelobacter models contain two sulfur reductase reactions SRE and SRE2 with hydrogen or NADPH as electron donors. To determine the stoichiometry of the proton translocation per pair of electrons transferred 


\begin{tabular}{|c|c|c|c|c|c|c|c|c|c|c|c|c|}
\hline \multirow{2}{*}{$\begin{array}{l}\text { Rate } \\
(\text { /h) }\end{array}$} & \multirow{2}{*}{\begin{tabular}{|c|} 
Initial \\
acetoin \\
$(\mathrm{mM})$
\end{tabular}} & \multirow{2}{*}{\begin{tabular}{|c|} 
Final \\
acetoin \\
$(\mathrm{mM})$ \\
\end{tabular}} & \multirow{2}{*}{\begin{tabular}{|c|} 
Final \\
acetate \\
$(\mathrm{mM})$
\end{tabular}} & \multirow{2}{*}{$\begin{array}{c}\text { Final } \\
\text { ethanol } \\
(\mathrm{mM})\end{array}$} & \multirow{2}{*}{$\begin{array}{l}\text { Protein } \\
(\mathrm{mg} / \mathrm{ml})\end{array}$} & \multirow[b]{2}{*}{ OD600 } & \multicolumn{6}{|c|}{$(\mathrm{mmol} / \mathrm{gdw} \mathrm{h})$} \\
\hline & & & & & & & qacetoin & qacetate & $\begin{array}{c}\text { qethanol } \\
\text { remaining } \\
\end{array}$ & $\begin{array}{c}\text { qethanol } \\
\text { produced }\end{array}$ & $\mathrm{qH}_{2}$ & ATPM \\
\hline 0.03 & 4.71 & 0.01 & 5.60 & 1.44 & 0166 & 0.138 & 3.907 & 655 & 1.197 & 2.704 & 1.602 & 3.704 \\
\hline 0.04 & 0 & 09 & 5.39 & 2.01 & 0.0164 & 0.134 & 5.172 & 6.047 & 2.255 & 3.691 & 1.892 & 4.779 \\
\hline 0.05 & 4.71 & 0.05 & 5.17 & 2.37 & 0.0160 & 0.132 & 6.699 & 7.432 & 3.407 & 5.208 & 1.643 & 5.847 \\
\hline 0.06 & 4.41 & 0.12 & 5.02 & 2.45 & \begin{tabular}{|l|}
0.0163 \\
\end{tabular} & 0.125 & 7.264 & 8.500 & 4.148 & 5.119 & 2.685 & 6.599 \\
\hline
\end{tabular}

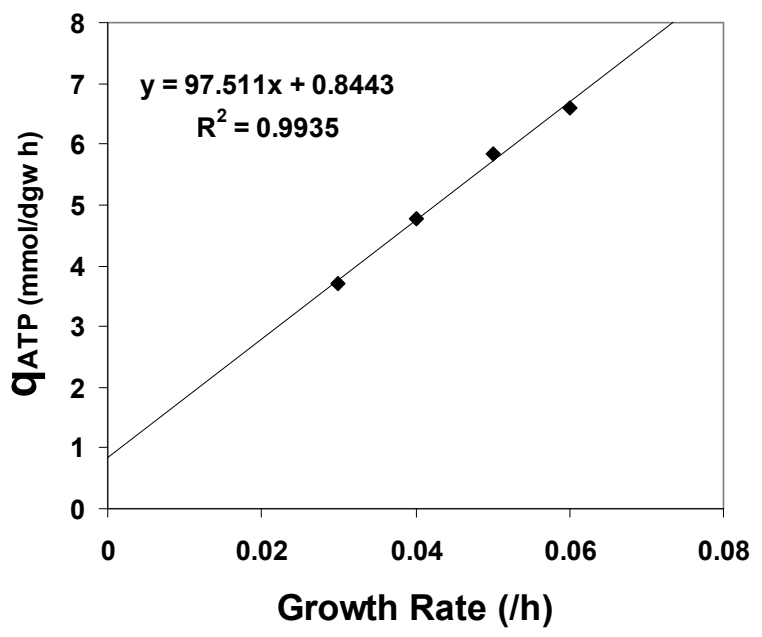

Figure 1 Determination of the energy parameters of the $P$. carbinolicus metabolic model. The table shows four sets of physiological data at different dilution rates for $P$. carbinolicus during acetoin fermentation in chemostats. The red experimental data were applied as constraints in simulations to obtain the predicted blue values, which were then used to generate the linear correlation graph to determine energy parameters of the $P$. carbinolicus metabolic model.

$\left(\mathrm{H}^{+} / 2 \mathrm{e}^{-}\right)$in the SRE and SRE2 two reactions, the published biomass yield data for $P$. carbinolicus grown in batch culture with ethanol or hydrogen as electron donor and Fe(III) as electron acceptor [17] were utilized. The biomass yield with ethanol as electron donor was compared to the biomass yield with hydrogen as electron donor, and the biomass yield ratios calculated from experimental results were compared to those from simulation results, where the $\mathrm{H}^{+} / 2 \mathrm{e}^{-}$ratio varied from 1 to 2 for SRE with hydrogen as electron donor or for SRE2 with ethanol as electron donor (Figure 2). As shown in Figure 2, simulation results with the $\mathrm{H}^{+} / 2 \mathrm{e}^{-}$ ratio of 2 for SRE and the $\mathrm{H}^{+} / 2 \mathrm{e}^{-}$ratio of 1 for SRE2 closely matched experimental results, whereas the other three combinations resulted in unmatched biomass yield ratios. Thus, the $\mathrm{H}^{+} / 2 \mathrm{e}^{-}$ratios were determined as 2 for SRE and 1 for SRE2 in P. carbinolicus model.

\section{Metabolic models of $P$. carbinolicus and $P$. propionicus}

Upon completion, the genome-scale $P$. carbinolicus metabolic model included 741 genes of the 3389 genes in the P. carbinolicus genome. As shown in Table 1, the

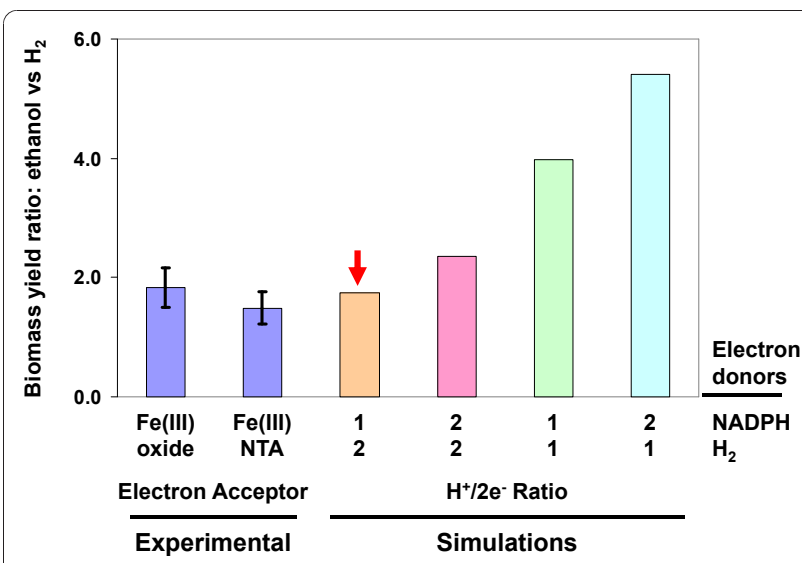

Figure 2 Determination of the stoichiometry of electron transfer in the two sulfur reductase reactions. The relative biomass yield ratios of ethanol to hydrogen were obtained from experimental data of $P$. carbinolicus grown in batch culture with ethanol or hydrogen as electron donor and Fe(III) oxide or Fe(III)NTA as electron acceptor [17], and were compared to simulation results varying the stoichiometry of the electron transfer in the two sulfur reductase reactions. The red arrow indicates a close match between experimental and simulation results. 
Table 1 Characteristics of the $P$. carbinolicus and $P$. propionicus genome-scale metabolic models compared with those of the $G$. sulfurreducens and $G$. metallireducens models

\begin{tabular}{|c|c|c|c|c|}
\hline & P. carbinolicus & P. propionicus & G. sulfurreducens & G. metallireducens \\
\hline Total Genes & 3389 & 3831 & 3468 & 3532 \\
\hline Included Genes & $741 \quad(21.9 \%)$ & $661(17.3 \%)$ & $712(20.5 \%)$ & $747 \quad(21.1 \%)$ \\
\hline Excluded Genes & 2648 (78.1\%) & 3170 (82.7\%) & $2756(79.5 \%)$ & $2785(78.9 \%)$ \\
\hline Total Proteins & 625 & 532 & 573 & 623 \\
\hline Total Reactions & 708 & 650 & 650 & 697 \\
\hline Non-gene Reactions & 37 (5.2\%) & $46 \quad(7.1 \%)$ & $34(5.2 \%)$ & $30(4.3 \%)$ \\
\hline Total Exchange Reactions & 69 & 53 & 58 & 61 \\
\hline Total Metabolites & 765 & 708 & 701 & 769 \\
\hline Extracellular Metabolites & $70 \quad(9.2 \%)$ & $52(7.3 \%)$ & 57 (8.1\%) & $60 \quad(7.8 \%)$ \\
\hline
\end{tabular}

P. carbinolicus model contains 708 reactions and 765 metabolites including 70 extracellular metabolites (see Additional File 2 for list of genes, reactions, and metabolites included in the P. carbinolicus metabolic model).

The genome-scale $P$. propionicus metabolic model included 661 genes among the 3831 genes in the P. propionicus genome. As shown in Table 1 , the P. propionicus model consists of 650 reactions and 708 metabolites including 52 extracellular metabolites (see Additional File 3 for list of genes, reactions, and metabolites included in the $P$. propionicus metabolic model).

As shown in Table 1, the Pelobacter models included about $20 \%$ of the genes in their genomes, similar to the Geobacter models [22,23]. The numbers of total reactions in the Pelobacter and Geobacter models are also comparable. Thus, these Geobacteraceae family members contain similar size genomes, share similar basic metabolic functions, and result in similar size genomescale metabolic models.

The reactions in both Pelobacter metabolic models were categorized according to 9 functional classifications (Figure 3). The two models share a similar distribution

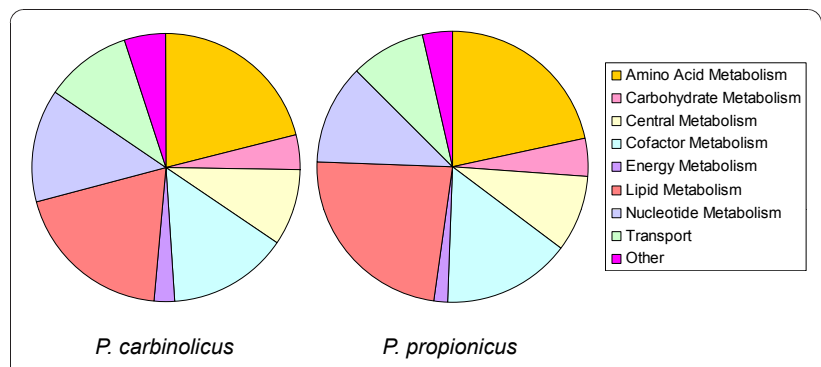

Figure 3 Functional classifications of reactions in the two Pelobacter metabolic models. Reactions in each Pelobacter metabolic model were categorized into 9 functional classifications. of reactions among different functional groups: reactions for metabolism of amino acids, lipids, cofactors, and nucleotides are the most abundant, accounting for almost $70 \%$ of all the reactions in both Pelobacter models.

Currently, there are 76 reactions associated with transporting metabolites in the $P$. carbinolicus model and 57 reactions for transporting metabolites in $P$. propionicus model, including redundant transporters for some extracellular metabolites. In addition, both Pelobacter genomes contained many genes related to ABC transport systems, which were not included in the network due to the lack of substrate specificity. Future experiments could provide additional evidence to include more transport systems.

\section{Validation of the metabolic models using published experimental data}

The Pelobacter metabolic models were validated using published experimental growth data. In cases where the experimental data was obtained from batch culture experiments where flux data was not readily available, experimentally determined growth rates were used to constrain the simulations. Ratios of biomass, substrate and product fluxes from model simulations were calculated and compared with the ratios from experimental value.

P. carbinolicus can grow by fermenting acetoin, 2,3butanediol, and ethylene glycol [6]. The P. carbinolicus metabolic model was validated by comparing experimental results with predicted results from in silico simulations of $P$. carbinolicus fermentative growth on these substrates (Figure 4). An optimal growth rate of $0.087 \mathrm{~h}^{-1}$ was observed with $P$. carbinolicus fermentative growth on 2,3-butanediol [6], and was applied to constrain all three simulations in which the substrate uptake rate was 


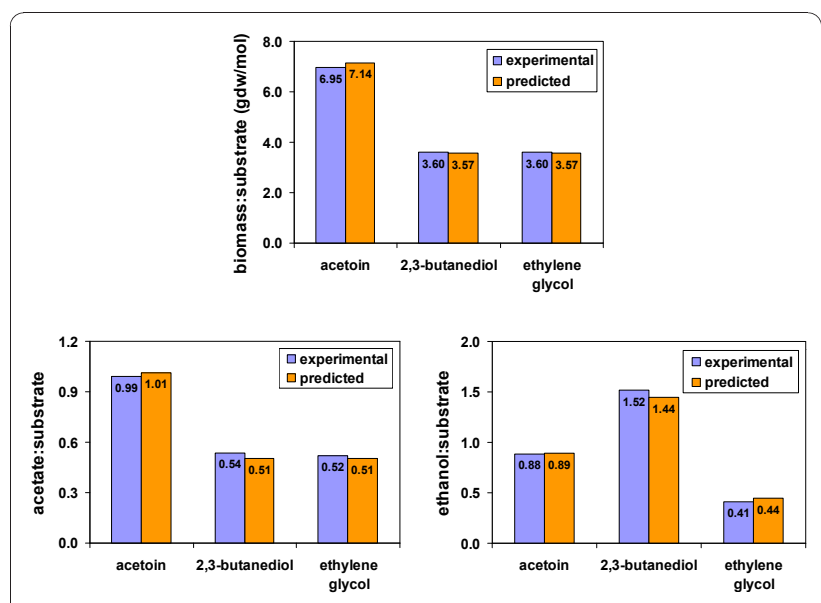

Figure 4 Validation of the $P$. carbinolicus metabolic model for fermentative growth. The biomass yield per mol of substrate, the ratios of produced acetate to substrates, and the ratios of produced ethanol to substrates were obtained from experimental data of $P$. carbinolicus fermenting acetoin, 2,3-butanediol, and ethylene glycol [6], and were compared to those predicted by in silico simulations with the $P$. carbinolicus metabolic model.

minimized. In simulations, the substrates acetoin, 2,3butanediol, and ethylene glycol were consumed to produce acetate and ethanol. The biomass yield per mole of acetoin was twice the biomass yield per mole of 2,3-butanediol and ethylene glycol in simulations, which matched well with the experimental data [6]. Similarly, the ratios of produced acetate and ethanol to consumed substrates predicted by in silico simulations were validated by the ratios from experimental results [6]. Thus, the fermentative growth of the $P$. carbinolicus metabolic model has been validated and can be applied in future modeling studies.

Similarly, the fermentative growth of the $P$. propionicus metabolic model was validated with experimental data obtained from $P$. propionicus fermentation of acetoin, 2,3-butanediol, ethanol, propanol, butanol, and lactate [6]. An optimal growth rate of $0.144 \mathrm{~h}^{-1}$ was observed with 2,3-butanediol fermentative growth [6], and was applied to constrain the simulations in which the substrate uptake rate was minimized. The predicted results from in silico simulations were compared with experimental results (Figure 5). In simulations, acetoin, 2,3butanediol, lactate, and ethanol were consumed to produce acetate and propionate, whereas substrates and acetate were both consumed to produce propionate in propanol and butanol fermentations. As shown in Figure 5 , the biomass yield per mole of acetoin was higher than the biomass yields per mole of 2,3-butanediol and lactate in simulations, and the latter two were much higher than the biomass yields per mole of the alcohols. The predicted biomass yields and ratios of acetate:substrate or propionate:substrate matched well with the experimental

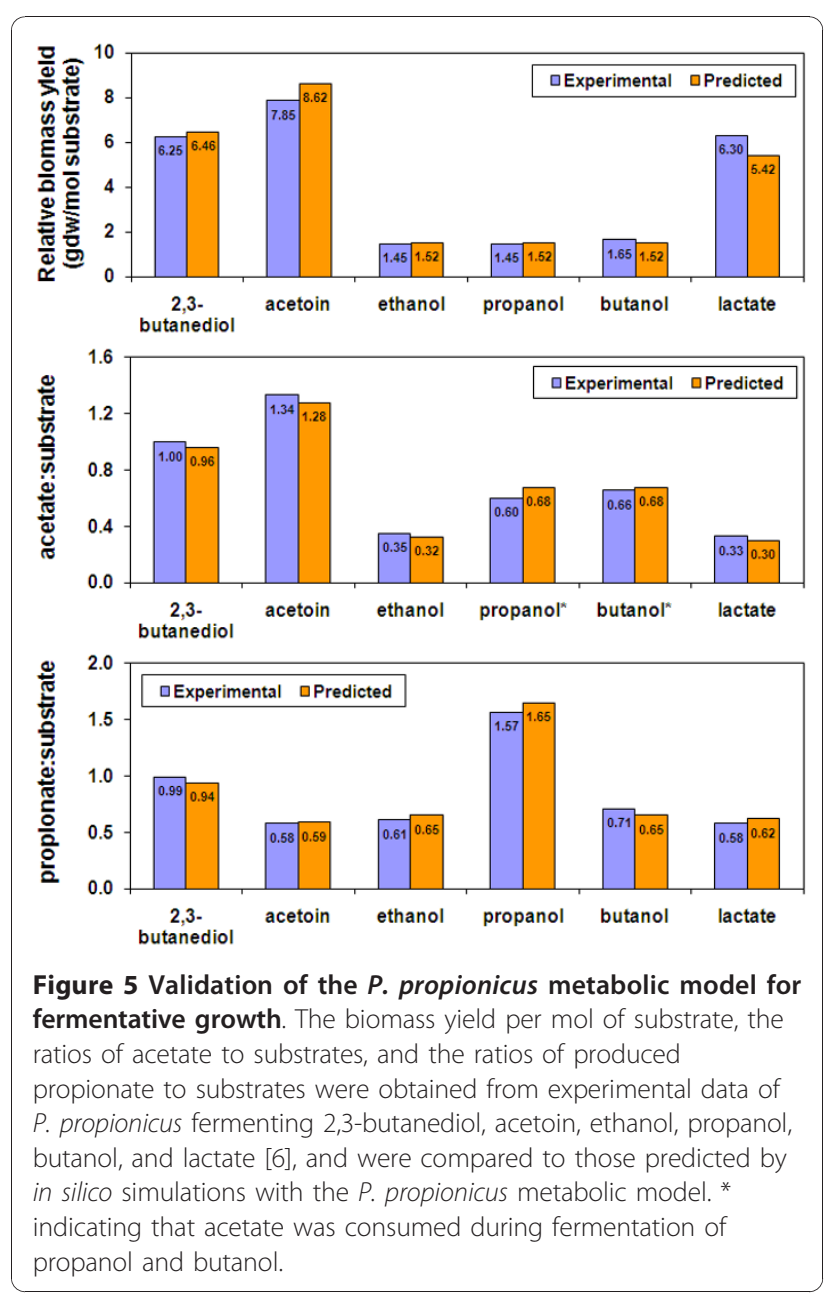

results. Therefore, the $P$. propionicus metabolic model has been validated with experimental fermentative growth results. In the $P$. propionicus metabolic model, lactate uptake was assumed to occur through a lactate proton antiporter for energy conservation and the simulated results predicted about $14 \%$ less biomass yield than the experimental results. Other lactate transporters such as a lactate proton symporter or diffusion were tested in simulations and resulted in much larger discrepancies with 38\%-63\% less biomass yields than the experimental results, supporting the assignment of a lactate proton antiporter for lactate uptake. However, further experimentation is required to confirm how lactate is transported.

$P$. carbinolicus can ferment ethanol to acetate with hydrogen generation in coculture with either Acetobacterium woodii or Methanospirillum hungatei as hydrogen utilizing partners [6]. Simulations of P. carbinolicus growth on 2,3-butanediol and ethanol with hydrogen production were conducted. The simulations predicted ratios of produced acetate to consumed substrates at 1.80 for acetate:2,3-butanediol and at 0.90 for acetate: 
ethanol, matched well with the experimental results at 1.96 for acetate:2,3-butanediol and at 0.93 for acetate: ethanol. The simulation results predicted a growth yield ratio of 2.0 for 2,3-butanediol to ethanol, close to the experimental growth yield ratio of 2.2 for 2,3-butanediol to ethanol. The P. carbinolicus model was validated for the hydrogen production for syntrophic growth with experimental results.

Like phylogenetically related Geobacter and Desulfuromonas species within the family Geobacteraceae, P. carbinolicus can utilize $\mathrm{Fe}(\mathrm{III})$ as the terminal electron acceptor [10], but sulfide is likely to serve as an electron shuttle for the Fe(III) reduction in P. carbinolicus [17]. P. carbinolicus grown on 2,3-butanediol with Fe(III) produced less ethanol and more acetate than grown on 2,3butanediol without $\mathrm{Fe}(\mathrm{III})$ [10]. The experimental acetate:ethanol ratios and growth rates were applied as constraints to simulate $\mathrm{Fe}(\mathrm{III})$ reduction by optimizing 2,3butanediol utilization. Simulation results of $P$. carbinolicus growth on 2,3-butanediol with or without $\mathrm{Fe}(\mathrm{III})$ are shown in Figure 6. The ratios of ethanol:2,3-butanediol and acetate:2,3-butanediol in the presence or absence of $\mathrm{Fe}$ (III) from in silico simulations matched well with those from the experimental data [10]. These results indicated that $P$. carbinolicus model worked well with $\mathrm{Fe}(\mathrm{III})$ reduction simulations.

P. carbinolicus is capable of utilizing $\mathrm{H}_{2}$ as the electron donor coupled with Fe(III) reduction to support growth when acetate is provided as the carbon source [10]. P. carbinolicus growth on ethanol or on hydrogen with acetate as the carbon source and with $\mathrm{Fe}(\mathrm{III})$ as the electron acceptor was simulated by applying experimental growth rates as constraints and optimizing the substrate utilization. As shown in Figure 7, the biomass yield ratios between ethanol/Fe(III) and $\mathrm{H}_{2} / \mathrm{Fe}$ (III) growth were calculated from both in silico result and experimental data, and were closely matched. Additionally, the relative ratios of acetate produced to $\mathrm{Fe}(\mathrm{III})$ reduced and ethanol consumed to $\mathrm{Fe}(\mathrm{III})$ reduced under
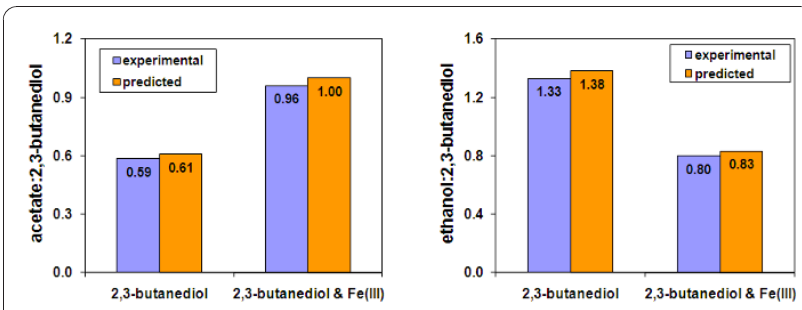

Figure 6 Validation of the $P$. carbinolicus metabolic model for growth with $\mathrm{Fe}$ (III) reduction. The ratios of produced acetate and ethanol to 2,3-butanediol were obtained from experimental data of P. carbinolicus grown on 2,3-butanediol with or without Fe(III) [10], and were compared to those predicted by in silico simulations with the $P$. carbinolicus metabolic model.

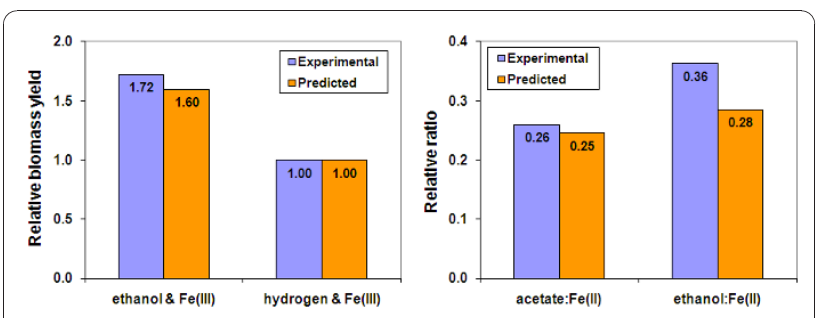

Figure 7 Validation of the $P$. carbinolicus metabolic model for growth utilizing hydrogen coupled with $\mathrm{Fe}$ (III) reduction. The relative biomass yield of ethanol to hydrogen and the ratios of acetate and ethanol to Fe(II) produced were obtained from experimental data of $P$. carbinolicus growth on hydrogen/acetate or ethanol with Fe(III) as the electron acceptor [10], and were compared to those predicted by in silico simulations with the $P$. carbinolicus metabolic model.

ethanol/Fe(III) growth conditions were similar between predicted and experimental results. The stoichiometry of $\mathrm{Fe}(\mathrm{III})$ reduced to hydrogen consumed was measured as 1.9 in experiments [10] and was calculated as 1.83 in simulations. These results were consistent between predicted and experimental values, and validated the $P$. carbinolicus model for $\mathrm{H}_{2}$ and ethanol utilization coupled with $\mathrm{Fe}(\mathrm{III})$ reduction.

\section{Comparison of the four Geobacteraceae metabolic models}

The four Geobacteraceae metabolic models were compared at the reaction level. The common reactions in all 4 models were determined for each functional category and the unique reactions in only one model but not in the other models were also determined (Table 2). The functional distribution of reactions among the four metabolic models shows similar patterns, with amino acid and lipid metabolism accounting for the most reactions and the energy metabolism category comprising the least reactions. Overall, 470 common reactions are present in all 4 models. These common reactions are largely distributed in functional categories including amino acid metabolism, cofactor metabolism, lipid metabolism, and nucleotide metabolism. Among the common reactions, the complete TCA cycle reactions are present in all 4 models, yet the Pelobacter species can not completely oxidize acetate and other organic electron donors to carbon dioxide [10] while the Geobacter species can. Another common reaction that is present in all 4 models is the nitrogenase reaction. The molybdenum nitrogenase enzyme complex contains the Fe protein encoded by the nifH gene and the MoFe protein encoded by the nifD and nifK genes [46,47], and the genes are well conserved among the four Geobacteraceae family members. The presence of the nitrogenase complex in all metabolic models suggested that all 4 species can fix nitrogen through ATP-dependent reduction of nitrogen to ammonia for growth, and it has 
Table 2 Comparison of reactions in the P. carbinolicus, P. propionicus, G. sulfurreducens, and G. metallireducens metabolic models

\begin{tabular}{|c|c|c|c|c|c|c|c|c|c|c|c|c|c|}
\hline & \multicolumn{3}{|c|}{ P. carbinolicus } & \multicolumn{3}{|c|}{ P. propionicus } & \multicolumn{3}{|c|}{ G. sulfurreducens } & \multicolumn{3}{|c|}{ G. metallireducens } & \multirow[t]{2}{*}{ Common } \\
\hline & All & Unique & $\%$ & All & Unique & $\%$ & All & Unique & $\%$ & All & Unique & $\%$ & \\
\hline Amino Acid Metabolism & 149 & 13 & $8.7 \%$ & 141 & 4 & $2.8 \%$ & 149 & 3 & $2.0 \%$ & 143 & 4 & $2.8 \%$ & 118 \\
\hline Carbohydrate Metabolism & 30 & 5 & $16.7 \%$ & 28 & 0 & $0.0 \%$ & 21 & 0 & $0.0 \%$ & 24 & 5 & $20.8 \%$ & 13 \\
\hline Central Metabolism & 65 & 6 & $9.2 \%$ & 60 & 6 & $10.0 \%$ & 56 & 1 & $1.8 \%$ & 62 & 5 & $8.1 \%$ & 39 \\
\hline Cofactor Metabolism & 103 & 3 & $2.9 \%$ & 99 & 2 & $2.0 \%$ & 106 & 1 & $0.9 \%$ & 104 & 0 & $0.0 \%$ & 93 \\
\hline Energy Metabolism & 16 & 6 & $37.5 \%$ & 11 & 1 & $9.1 \%$ & 22 & 4 & $18.2 \%$ & 20 & 1 & $5.0 \%$ & 8 \\
\hline Lipid Metabolism & 138 & 8 & $5.8 \%$ & 153 & 2 & $1.3 \%$ & 130 & 4 & $3.1 \%$ & 159 & 18 & $11.3 \%$ & 98 \\
\hline Nucleotide Metabolism & 96 & 24 & $25.0 \%$ & 77 & 0 & $0.0 \%$ & 77 & 0 & $0.0 \%$ & 76 & 2 & $2.6 \%$ & 65 \\
\hline Transport & 76 & 19 & $25.0 \%$ & 57 & 1 & $1.8 \%$ & 62 & 10 & $16.1 \%$ & 62 & 22 & $35.5 \%$ & 21 \\
\hline Other & 35 & 8 & $22.9 \%$ & 24 & 1 & $4.2 \%$ & 27 & 2 & $7.4 \%$ & 47 & 22 & $46.8 \%$ & 15 \\
\hline Total reactions & 708 & 92 & $13.0 \%$ & 650 & 17 & $2.6 \%$ & 650 & 25 & $3.8 \%$ & 697 & 79 & $11.3 \%$ & 470 \\
\hline
\end{tabular}

shown that G. sulfurreducens can grow by nitrogen fixation [48].

Although the four species shared many common reactions among their metabolic models, each model contains some unique reactions, reflecting the unique metabolic capabilities of the Geobacteraceae species. As shown in Table 2, the $P$. carbinolicus model contains 92 unique reactions and the G. metallireducens model contains 79 unique reactions, whereas the P. propionicus model contains only 17 unique reactions and the G. sulfurreducens model contains 25 unique reactions. For the $P$. carbinolicus model, these unique reactions include, for example: ethylene glycol dehydratase for the fermentation of ethylene glycol; purine-nucleoside phosphorylase for the formation of purines like adenosine, deoxyadenosine, xanthosine, and hypoxanthine; and acetylornithine deacetylase and ornithine cyclodeaminase to form a second pathway for proline biosynthesis through L-ornithine in addition to the common proline biosynthesis pathway through 1-pyrroline-5-carboxylate. Additionally, $P$. carbinolicus contains the pyruvate formate lyase and formate dehydrogenase reactions that can convert pyruvate to acetyl-CoA with the co-production of hydrogen for syntrophic growth with hydrogenconsuming organisms. The unique reactions in the $P$. propionicus model include the reactions for the methylmalonyl-CoA dependent propionate formation pathway. The citrate lyase reaction is also unique to the $P$. propionicus model but its physiological role is not clear yet. See Additional File 4 for a list of unique reactions in the four Geobacteraceae metabolic models.

\section{Incorporation of gene expression levels in metabolic models}

The reconstructed metabolic models contain duplicate genes for the same reaction, or alternative reactions between two metabolites, or alternative pathways among metabolites. Without additional constraints, the duplicate genes, alternative reactions, or alternative pathways can all direct metabolic fluxes in simulations. Gene expression data suggesting the preference of duplicate genes, alternative reactions, or alternative pathways can be utilized to constrain the simulations and improve computational modeling analysis. On the other hand, the metabolic models can also be utilized to facilitate the interpretation of gene expression data. The $P$. carbinolicus metabolic model and the microarray data obtained from $P$. carbinolicus cells cultured by acetoin fermentation or ethanol/Fe(III) respiration [17] were compared.

During acetoin fermentation, acetoin is degraded into acetaldehyde and acetyl-CoA by the acetoin:DCPIP oxidoreductase. When the gene expression levels were displayed on the $P$. carbinolicus metabolic map, genes for the acetoin:DCPIP oxidoreductase Pcar_0343-0347 were among the most highly transcribed genes during acetoin fermentation. During ethanol/Fe(III) respiration, the expression levels of these genes decreased more than 10 fold [17]. Genes with the highest transcription levels during ethanol/Fe(III) respiration include Pcar_0251 and Pcar_0255 for the alcohol dehydrogenase (ethanol:NAD) reaction and Pcar_2758 for the acetaldehyde dehydrogenase reaction, involved in the oxidation of ethanol to acetyl-CoA[17]. These genes were downregulated more than 5 fold during acetoin fermentation compared to ethanol/Fe(III) respiration [17]. The metabolic map and model added an additional tool to understand the gene expression data from a metabolic point of view.

The metabolic model contains redundancy in the form of duplicate genes, alternative reactions, or alternative pathways. Such redundancy may play important roles in genetic and metabolic robustness. Microarray analysis 
indicated that this redundancy was tightly regulated in P. carbinolicus. For example, two acetyl-CoA:phosphate acetyltransferase isozymes Pcar_2542 and Pcar_2850 are present in the $P$. carbinolicus model. The gene expression level of Pcar_2542 was 23 fold more than the level of Pcar_2850 during acetoin fermentation, suggesting that Pcar_2542 was the primary enzyme to catalyze the reaction in acetoin fermentation. Similar examples included isozymes for aconitase, acetate kinase, acetoin dehydrogenase, acetaldehyde dehydrogenase, etc.

When different reactions for the same metabolite are present, it is likely for the cell to use the most energy efficient reaction for optimal growth. An example of this is the presence of a sulfate proton symport transporter and a sulfate $A B C$ transport system for sulfate transport. The expression level of the proton symport transporter gene Pcar_0676 was more than the sulfate ABC transport system genes during acetoin fermentation (expression of Pcar_0676 was about 8 times Pcar_2084). Thus, it was likely that sulfate was mainly transported through the proton symport transporter during acetoin fermentation. Similarly, the genes encoding nitrogen fixation enzymes were turned off as the cell used ammonium from the culture medium. The P. carbinolicus metabolic model contains a biosynthesis pathway for proline from glutamate through ornithine, in addition to the 1-pyrroline-5-carboxylate-dependent pathway present in the G. sulfurreducens model. Microarray results indicated that the mRNA levels of pyrroline-5-carboxylate reductase and ornithine cyclodeaminase were similar under both acetoin fermentation and ethanol/Fe(III) respiration conditions, suggesting that both pathways were used for proline biosynthesis.

P. carbinolicus contains genes for de novo cobalamin biosynthesis, but some of these genes were among the lowest expression levels during both culture conditions. Most likely, $P$. carbinolicus did not synthesize cobalamin, but utilized the vitamin $B_{12}$ supplemented in the culture medium. When compared to the G. sulfurreducens model, P. carbinolicus had some unique reactions allowing the formation of purines like adenosine, inosine, deoxyinosine, xanthine, hypoxanthine, and urate. However, gene expression data suggested that only adenosine was formed during both culture conditions, whereas the others were unlikely to be synthesized.

The microarray results will be utilized to constrain the reconstructed network, where the reactions are closed during simulations when the associated genes have very low expression level for the corresponding culture conditions.

\section{TCA cycle and sulfur reduction}

P. carbinolicus reduces $\mathrm{Fe}(\mathrm{III})$ indirectly through sulfur reduction [17]. However, P. carbinolicus only incompletely oxidizes organic substrates to acetate using $\mathrm{Fe}(\mathrm{III})$ as an electron acceptor [10]. The complete TCA cycle reactions are present in $P$. carbinolicus model, yet the fully functional TCA cycle was not observed during simulations with $\mathrm{Fe}(\mathrm{III})$ as electron acceptor. Further examination suggested that this was due to the inability in coupling succinate oxidation to sulfur reduction $\left(\Delta \mathrm{G}_{0}{ }^{\prime}=+53 \mathrm{~kJ} / \mathrm{mol}\right)$ [49]. Many archaea and bacteria are able to reduce elemental sulfur using hydrogen or organic substrates as electron donors [50]. However, only a few bacteria can completely oxidize acetate with sulfur reduction, including Desulfuromonas acetoxidans utilizing an ATP-driven succinate oxidation mechanism for acetate oxidation to $\mathrm{CO}_{2}$ with elemental sulfur as electron acceptor [49,51]. It seems that $P$. carbinolicus does not contain such an ATPdriven succinate oxidation mechanism, thus cannot completely oxidize acetate to $\mathrm{CO}_{2}$ with elemental sulfur or $\mathrm{Fe}$ (III) as electron donor.

\section{Implications}

In subsurface environments, Pelobacter species can be closely associated with Geobacter species $[19,20]$. The Pelobacter and Geobacter metabolic models share 470 common reactions, about $70 \%$ of all reactions in the models. Genome sequence analysis of six Geobacteraceae family members including all four species discussed in this study suggested that the two Pelobacter species evolved separately with fermentative/syntrophic metabolism from a common Geobacteraceae ancestor with anaerobic respiratory metabolism [8]. The P. carbinolicus model contains reactions for fermentative growth with various organic substrates and reactions of hydrogenase and formate dehydrogenase to produce hydrogen and formate for syntrophic growth with hydrogen- and formate-consuming organisms. The $P$. propionicus model contains reactions for fermentative growth with different organic substrates producing propionate. The Pelobacter models contain metabolic abilities reflecting their physiological roles in the subsurface community. The evolution of the Pelobacter species to fermentative metabolism could also result in the loss of many $c$-type cytochromes from their genomes, since there is no need to transfer electrons outside the cells during fermentative growth, thus abolishing their abilities to directly reduce $\mathrm{Fe}(\mathrm{III})$ or the anodes of microbial fuel cells. Furthermore, the evolution of a subsurface community containing fermentative and respiratory species allowing the breakdown of complex organic substrates to $\mathrm{CO}_{2}$ through different species may be advantageous as each species does not need to synthesize a full set of proteins for the complete oxidization of complex organic substrates. The development of the metabolic models of these Geobacteraceae species will facilitate the study of their roles and interactions in the subsurface microbial community. 


\section{Conclusions}

We have developed genome-scale metabolic models of $P$. carbinolicus and P. propionicus. These models of Pelobacter metabolism can now be incorporated into the growing repertoire of genome scale models of the Geobacteraceae family to aid in describing the growth and activity of these organisms in anoxic environments and in the study of their roles and interactions in the subsurface microbial community.

\section{Additional material}

Additional file 1: Metabolic maps for the $P$. carbinolicus and $P$. propionicus genome-scale metabolic models.

Additional file 2: All the reactions, genes, and metabolites included in the $P$. carbinolicus genome-scale metabolic model.

Additional file 3: All the reactions, genes, and metabolites included in the $P$. propionicus genome-scale metabolic model.

Additional file 4: All the unique reactions in each of the four Geobacteraceae genome-scale metabolic models.

\section{List of abbreviations}

DCPIP: 2,6-dichlorophenolindophenol; GAM: growth-associated maintenance; gdw: gram dry weight; GPR: gene-protein-reaction; HPLC: high-pressure liquid chromatography; nGAM: non-growth associated maintenance; ORF: open reading frame.

\section{Acknowledgements}

This research was supported by the Office of Science (BER), Genomics:GTL Program of the U.S. Department of Energy, Grant No. DE-FC02-02ER63446.

\section{Author details}

${ }^{1}$ Genomatica Inc., 10520 Wateridge Circle, San Diego, CA, USA. ²Department of Microbiology, University of Massachusetts, Amherst, MA, USA. ${ }^{3}$ Luca Technologies Inc., 500 Corporate Circle, Golden, CO, USA. ${ }^{4}$ Pathway Genomics Corporation, 4045 Sorrento Valley Blvd., San Diego, CA, USA.

\section{Authors' contributions}

JS, OB, and TRF developed the genome-scale metabolic models of $P$. carbinolicus and $P$. propionicus. SAH carried out the growth experiments. IS analyzed the experimental data and drafted the manuscript. JS, SAH, and DRL conceived the study and revised the manuscript. All authors read and approved the final manuscript.

Received: 13 August 2010 Accepted: 23 December 2010 Published: 23 December 2010

\section{References}

1. Narasingarao P, Haggblom MM: Identification of anaerobic selenaterespiring bacteria from aquatic sediments. Appl Environ Microbiol 2007, 73:3519-3527.

2. Mussmann M, Ishii $K$, Rabus $R$, Amann R: Diversity and vertical distribution of cultured and uncultured Deltaproteobacteria in an intertidal mud flat of the Wadden Sea. Environmental Microbiology 2005, 7:405-418.

3. Chauhan A, Ogram A: Phylogeny of acetate-utilizing microorganisms in soils along a nutrient gradient in the Florida Everglades. Appl Environ Microbiol 2006, 72:6837-6840.

4. Shimizu S, Akiyama M, Naganuma T, Fujioka M, Nako M, Ishijima Y: Molecular characterization of microbial communities in deep coal seam groundwater of northern Japan. Geobiology 2009, 5:423-433.

5. Dahle H, Garshol F, Madsen M, Birkeland NK: Microbial community structure analysis of produced water from a high-temperature North Sea oil-field. Antonie Van Leeuwenhoek 2008, 93:37-49.
6. Schink B: Fermentation of 2,3-Butanediol by Pelobacter-Carbinolicus SpNov and Pelobacter-Propionicus Sp-Nov, and Evidence for Propionate Formation from C-2 Compounds. Archives of Microbiology 1984, 137:33-41.

7. Lonergan DJ, Jenter HL, Coates JD, Phillips EJP, Schmidt TM, Lovley DR: Phylogenetic analysis of dissimilatory Fe(III)-reducing bacteria. Journal of Bacteriology 1996, 178:2402-2408.

8. Butler JE, Young ND, Lovley DR: Evolution from a respiratory ancestor to fill syntrophic and fermentative niches: comparative fenomics of six Geobacteraceae species. BMC Genomics 2009, 10:103.

9. Holmes DE, Nevin KP, Lovley DR: Comparison of $16 \mathrm{~S}$ rRNA, nifD, recA, gyrB, rpoB and fusA genes within the family Geobacteraceae fam. nov. Int J Syst Evol Microbiol 2004, 54:1591-1599.

10. Lovley DR, Phillips EJ, Lonergan DJ, Widman PK: Fe(III) and So reduction by Pelobacter carbinolicus. Appl Environ Microbiol 1995, 61:2132-2138.

11. Lovley DR: Dissimilatory Fe(III) and Mn(IV) reduction. Microbiol Rev 1991, 55:259-287

12. Haveman SA, Holmes DE, Ding YHR, Ward JE, DiDonato RJ, Lovley DR: CType Cytochromes in Pelobacter carbinolicus. App/ Environ Microbiol 2006, 72:6980-6985.

13. Butler JE, Kaufmann F, Coppi MV, Nunez C, Lovley DR: MacA a diheme ctype cytochrome involved in Fe(III) reduction by Geobacter sulfurreducens. Journal of Bacteriology 2004, 186:4042-4045.

14. Leang C, Coppi MV, Lovley DR: OmcB, a c-type polyheme cytochrome, involved in Fe(III) reduction in Geobacter sulfurreducens. J Bacteriol 2003, 185:2096-2103.

15. Lloyd JR, Leang C, Hodges Myerson AL, Coppi MV, Cuifo S, Methe B, et al: Biochemical and genetic characterization of PpcA, a periplasmic c-type cytochrome in Geobacter sulfurreducens. Biochem J 2003, 369:153-161.

16. Mehta T, Coppi MV, Childers SE, Lovley DR: Outer membrane c-type cytochromes required for $\mathrm{Fe}(\mathrm{III})$ and $\mathrm{Mn}$ (IV) oxide reduction in Geobacter sulfurreducens. Appl Environ Microbiol 2005, 71:8634-8641.

17. Haveman SA, Didonato RJ, Villanueva L, Shelobolina ES, Postier BL, Xu B, et al: Genome-wide gene expression patterns and growth requirements suggest that Pelobacter carbinolicus reduces Fe(III) indirectly via sulfide production. Appl Environ Microbiol 2008, 74:4277-4284.

18. Richter H, Lanthier M, Nevin KP, Lovley DR: Lack of Electricity Production by Pelobacter carbinolicus Indicates that the Capacity for Fe(III) Oxide Reduction does Not Necessarily Confer the Ability for Electron Transfer to Fuel Cell Anodes. Appl Environ Microbiol 2007, 73:5347-5353.

19. Holmes DE, Bond DR, O'Neil RA, Reimers CE, Tender LR, Lovley DR: Microbial communities associated with electrodes harvesting electricity from a variety of aquatic sediments. Microb Ecol 2004, 48:178-190.

20. Vrionis HA, Anderson RT, Ortiz-Bernad I, O'Neill KR, Resch CT, Peacock AD, et al: Microbiological and geochemical heterogeneity in an in situ uranium bioremediation field site. Appl Environ Microbiol 2005, 71:6308-6318.

21. Lovley DR: Cleaning up with genomics: applying molecular biology to bioremediation. Nat Rev Microbiol 2003, 1:35-44.

22. Mahadevan R, Bond DR, Butler JE, Esteve-Nunez A, Coppi MV, Palsson BO, et al: Characterization of metabolism in the Fe(III)-reducing organism Geobacter sulfurreducens by constraint-based modeling. Appl Environ Microbiol 2006, 72:1558-1568.

23. Sun J, Sayyar B, Butler JE, Pharkya P, Fahland TR, Famili I, et al: Genomescale constraint-based modeling of Geobacter metallireducens. BMC Syst Biol 2009, 3:15.

24. Izallalen M, Mahadevan R, Burgard A, Postier B, Didonato R Jr, Sun J, et al: Geobacter sulfurreducens strain engineered for increased rates of respiration. Metab Eng 2008, 10:267-275.

25. Segura D, Mahadevan R, Juarez K, Lovley DR: Computational and experimental analysis of redundancy in the central metabolism of Geobacter sulfurreducens. PLoS Comput Biol 2008, 4:e36.

26. Butler JE, He Q, Nevin KP, He Z, Zhou J, Lovley DR: Genomic and microarray analysis of aromatics degradation in Geobacter metallireducens and comparison to a Geobacter isolate from a contaminated field site. BMC Genomics 2007, 8:180.

27. Risso C, Van Dien SJ, Orloff A, Lovley DR, Coppi MV: Elucidation of an alternate isoleucine biosynthesis pathway in Geobacter sulfurreducens. J Bacteriol 2008, 190:2266-2274.

28. Krushkal J, Yan B, DiDonato LN, Puljic M, Nevin KP, Woodard TL, et al: Genome-wide expression profiling in Geobacter sulfurreducens: 
identification of Fur and RpoS transcription regulatory sites in a relGsu mutant. Funct Integr Genomics 2007, 7:229-255.

29. Mahadevan R, Lovley DR: The degree of redundancy in metabolic genes is linked to mode of metabolism. Biophys J 2008, 94:1216-1220.

30. Smith PK, Krohn Rl, Hermanson GT, Mallia AK, Gartner FH, Provenzano MD, et al: Measurement of protein using bicinchoninic acid. Anal Biochem 1985, 150:76-85.

31. Price ND, Reed JL, Palsson BO: Genome-scale models of microbial cells: evaluating the consequences of constraints. Nat Rev Microbiol 2004, 2:886-897.

32. Price ND, Papin JA, Schilling $\mathrm{CH}$, Palsson BO: Genome-scale microbial in silico models: the constraints-based approach. Trends Biotechnol 2003, 21:162-169.

33. Teusink B, Wiersma A, Molenaar D, Francke C, de Vos WM, Siezen RJ, et al: Analysis of growth of Lactobacillus plantarum WCFS1 on a complex medium using a genome-scale metabolic model. J Biol Chem 2006, 281:40041-40048.

34. Edwards JS, Palsson BO: The Escherichia coli MG1655 in silico metabolic genotype: its definition, characteristics, and capabilities. Proc Natl Acad Sci USA 2000, 97:5528-5533.

35. Oh YK, Palsson BO, Park SM, Schilling CH, Mahadevan R: Genome-scale reconstruction of metabolic network in bacillus subtilis based on highthroughput phenotyping and gene essentiality data. J Biol Chem 2007, 282:28791-28799.

36. Oppermann FB, Steinbuchel A, Schlegel HG: Utilization of Methylacetoin by the Strict Anaerobe Pelobacter-Carbinolicus and Consequences for the Catabolism of Acetoin. Fems Microbiology Letters 1988, 55:47-52.

37. Oppermann FB, Steinbuchel A: Identification and Molecular Characterization of the Aco Genes Encoding the Pelobacter-Carbinolicus Acetoin Dehydrogenase Enzyme-System. Journal of Bacteriology 1994, 176:469-485

38. Oppermann FB, Schmidt B, Steinbuchel A: Purification and Characterization of Acetoin-2,6-Dichlorophenolindophenol Oxidoreductase, Dihydrolipoamide Dehydrogenase, and Dihydrolipoamide Acetyltransferase of the Pelobacter-Carbinolicus Acetoin Dehydrogenase Enzyme-System. Journal of Bacteriology 1991, 173:757-767.

39. Schink B, Kremer DR, Hansen TA: Pathway of Propionate Formation from Ethanol in Pelobacter-Propionicus. Archives of Microbiology 1987, 147:321-327.

40. Seeliger S, Janssen PH, Schink B: Energetics and kinetics of lactate fermentation to acetate and propionate via methylmalonyl-CoA or acrylyl-CoA. FEMS Microbiol Lett 2002, 211:65-70.

41. Straub KL, Schink B: Ferrihydrite-dependent growth of Sulfurospirillum deleyianum through electron transfer via sulfur cycling. Appl Environ Microbiol 2004, 70:5744-5749.

42. Nelson DL, Cox MM: Lehninger Principles of Biochemistry. 3 edition. New York, NY: Worth Publishers; 2000.

43. Laska S, Lottspeich F, Kletzin A: Membrane-bound hydrogenase and sulfur reductase of the hyperthermophilic and acidophilic archaeon Acidianus ambivalens. Microbiology 2003, 149:2357-2371.

44. Ng KY, Sawada R, Inoue S, Kamimura K, Sugio T: Purification and some properties of sulfur reductase from the iron-oxidizing bacterium Thiobacillus ferrooxidans NASF-1. J Biosci Bioeng 2000, 90:199-203.

45. Feist AM, Henry CS, Reed JL, Krummenacker M, Joyce AR, Karp PD, et al: A genome-scale metabolic reconstruction for Escherichia coli K-12 MG1655 that accounts for 1260 ORFs and thermodynamic information. Mol Syst Biol 2007, 3:121.

46. Rubio LM, Ludden PW: Biosynthesis of the iron-molybdenum cofactor of nitrogenase. Annu Rev Microbiol 2008, 62:93-111.

47. Hu Y, Fay AW, Lee CC, Yoshizawa J, Ribbe MW: Assembly of nitrogenase MoFe protein. Biochemistry 2008, 47:3973-3981.

48. Methe BA, Webster J, Nevin K, Butler J, Lovley DR: DNA microarray analysis of nitrogen fixation and $\mathrm{Fe}$ (III) reduction in Geobacter sulfurreducens. Appl Environ Microbiol 2005, 71:2530-2538.

49. Paulsen J, Kroger A, Thauer RK: ATP driven succinate oxidation in the catabolism of Desulfuromonas acetoxidans. Arch Microbiol 1986, 144:78-83.

50. Hedderich R, Klimmek O, Kroger A, Dirmeier R, Keller M, Stetter KO: Anaerobic respiration with elemental sulfur and with disulfides. FEMS Microbiol Rev 1999, 22:353-381.
51. Gebhardt NA, Thauer RK, Linder D, Kaulfers PM, Pfennig N: Mechanism of acetate oxidation to carbon dioxide with elemental sulfur in Desulfuromonas acetoxidans. Arch Microbiol 1985, 141:392-398.

doi:10.1186/1752-0509-4-174

Cite this article as: Sun et al:: Constraint-based modeling analysis of the metabolism of two Pelobacter species. BMC Systems Biology 2010 4:174.

\section{Submit your next manuscript to BioMed Central and take full advantage of:}

- Convenient online submission

- Thorough peer review

- No space constraints or color figure charges

- Immediate publication on acceptance

- Inclusion in PubMed, CAS, Scopus and Google Scholar

- Research which is freely available for redistribution

Submit your manuscript at www.biomedcentral.com/submit
C Biomed Central 